\title{
MORPHOLOGIC SPECTRUM AND CLINICOPATHOLOGICAL CORRELATION OF GASTROINTESTINAL STROMAL TUMOURS: AN EXPERIENCE OF 6 YEARS AT A TERTIARY CARE HOSPITAL
}

\author{
Asim Qureshi, Hina Tariq, Zafar Ali, Nadira Mamoon, Imran N. Ahmed, Asna H. Khan, \\ Humaira Nasir \\ Department of Pathology, Shifa International Hospital, Islamabad, Pakistan \\ Received: 14 September 2016 / Accepted: 19 December 2016
}

\begin{abstract}
Objective: The objective of this study was to determine the morphologic spectrum and risk category of gastrointestinal stromal tumour (GIST) and compare with overall patient survival.

Materials and Methods: It is a descriptive observational study. The study was carried at Shifa International Hospital, Islamabad. Duration of the study was from January 2009 to January 2015. A total of 31 patients with the diagnosis of GIST were included, irrespective of age and gender. Data were retrieved from laboratory information system. Results were analysed by statistical software, Statistical Package of the Social Sciences. Morphologic type, site of tumour, risk category and overall survival were determined and mean, standard deviation, frequencies and percentages were calculated for age site and risk category.
\end{abstract}

Results: Of 31 patients, $21(67.7 \%)$ were male and $10(32.3 \%)$ were female. Site of tumour was as follows: Gastric $13(41.9 \%)$, extra visceral $6(19.4 \%)$, small intestine $9(29.0 \%)$, rectum $2(6.5 \%)$ and pancreas $1(3.2 \%)$. According to risk categorisation, one was categorised as (3.2\%) very low risk, 3 (9.7\%) low risk, 5 (16.1\%) intermediate risk and $22(71 \%)$ high risk. Follow-up was available in 21 patients. 7 patients (22.5\%) lost to follow-up. 8 (25\%) had recurrence and 4 (12.9\%) died.

Conclusion: Majority of cases diagnosed at our centre were gastric in origin followed by small intestine, and as per risk categorisation, most were high risk. Patient survival with high-risk tumours was dismal.

Key words: Gastrointestinal stromal tumour, immunohistochemistry, risk categorisation

\section{Introduction}

Gastrointestinal stromal tumours (GISTs) were initially believed to be of smooth muscle origin. ${ }^{[1]}$ However, now, this theory has been replaced by the fact that these originate from cells of Cajal, the pacemakers of gut. ${ }^{[2]}$ Many population-based studies in Western countries have been published regarding epidemiology and prognosis of GISTs. However, there are few studies available in literature to document the characteristics of GISTs in Asian countries, especially in Pakistan. ${ }^{[3]}$

GIST-affected patients have vague symptoms and mostly are discovered incidentally. ${ }^{[1,3,4]}$ Most GISTs arise

$\overline{\text { Correspondence: Dr. Asim Qureshi, Department of Pathology, Shifa }}$ International Hospital, Islamabad, Pakistan.

Email: asimqureshi32@gmail.com sporadically, but a small proportion arises with other tumours, (Carney triad) familial GIST syndrome and neurofibromatosis type 1 (NF1). ${ }^{[4]}$

Stomach is the most common site of GIST (approximately $60-70 \%)$. Followed by small intestine (20-30\%), while $<10 \%$ arise from oesophagus, colon, rectum, omentum and mesentery. Around $10-30 \%$ of GISTs fall in high-risk category. Omentum/peritoneum and liver are the most common sites of recurrence and metastasis. ${ }^{[2]}$

The diagnosis of GIST is made on typical histologic features; however, it has to be confirmed by immunohistochemical (IHC) panel including CD117 (c-kinase receptor [Kit]) and diagnosed on GIST (DOG1). CD 117 positivity is important for diagnosis of GIST as well as for 
therapeutic reasons. Up to $95 \%$ are positive for CD $117 .{ }^{[2]}$ About $70 \%$ of GIST are positive for CD34. The current recommendation by the College of American Pathologists cancer protocols, regarding the behaviour of GIST, is their stratification into very low, low, intermediate, high and very high-risk groups. This grouping is based on assessing location of tumour, gross tumour size as well as mitotic count in 20 high-power fields (HPFs) Armed Forces Institute of Pathology criteria for risk assessment. ${ }^{[2,5,6]}$

Majority (85\%) of GISTs have mutations in the gene encoding the transmembrane tyrosine kinase receptor (KIT). Most GISTs which lack KIT mutations are wildtype (approximately 10\%) or harbour mutations in platelet-derived growth factor receptor which is highly homologous with KIT. NF1-associated GISTs usually retain expression of succinate dehydrogenase. ${ }^{[4]}$

Majority (about 80\%) of gastric GISTs have low risk for malignancy. Duodenal GISTs are most often found in the second part of duodenum and about half of them have high risk for malignancy. GISTs of colon, anorectum, oesophagus, omentum and peritoneum represent a small percentage of all GISTs. Therefore, less is known about their biologic behaviour. ${ }^{[2,7,8]}$

While evaluating excision specimen of GIST, clinical history and any neoadjuvant treatment details are essential. Prior morphological appearance (spindle vs. epithelioid) and CD117 expression should also be known, as this information may change treatment. ${ }^{[2,7,9]}$

\section{Materials and Methods}

A total of 31 patients who underwent excision of GIST from January 2009 to January 2015, at Shifa International Hospital were evaluated, after approval from the institution research board and ethical committee. Cases were included, irrespective of age, gender and with or without neoadjuvant chemotherapy administration.

The data were analysed by SPSS 17 . Mean \pm standard deviation (SD) of quantitative variables like age was calculated. Frequency and percentage for variables such as gender, tumour site, tumour size, mitotic count, morphologic pattern, necrosis and risk categorisation were determined. IHC pattern of CD117, DOG1,
ASMA, CKAE1/AE3 and S100, resection margin status, neoadjuvant chemotherapy administration and its effects were noted.

\section{Results}

Of 31 patients, $21(67.7 \%)$ were male and $10(32.3 \%)$ were female with male-to-female ratio of 2.1:1. Distribution according to the location of tumour was as follows: Gastric $13(41.9 \%)$, extra visceral $6(19.4 \%)$, small intestine $9(29.0 \%)$, rectum $2(6.5 \%)$ and pancreas 1 (3.2\%). In cases of small intestine, jejunum/ileum was the site in $3(9.7 \%)$ and duodenum in $2(6.5 \%)$, while in $4(12.9 \%)$ exact site in small intestine was not specified.

Tumour was $<5 \mathrm{~cm}$ in 6 cases $(19.4 \%),>5 \mathrm{~cm}$ but $<10 \mathrm{~cm}$ in 12 cases (38.7\%) and $>10 \mathrm{~cm}$ in $13(41.9 \%)$ cases. Mitotic figures per $50 \mathrm{HPF}$ were $\leq 5$ in 11 cases $(35.5 \%)$ and $>5$ in 20 cases $(64.5 \%)$. According to risk categorisation, 1 was $(3.2 \%)$ very low risk, 3 (9.7\%) low risk, $5(16.1 \%)$ intermediate risk and 22 (71\%) high risk [Table 1].

Of 31 cases, $21(67.7 \%)$ had spindle cell morphology, $7(22.58 \%)$ had epithelioid appearance and $2(6 \%)$ had mixed morphology. Necrosis was present in $48.4 \%$ $(n=15)$. Tumour was unifocal in $30(96.8 \%)$ cases and multifocal in $1(3.2 \%)$ cases. $16(51.6 \%)$ had free resection margins.

CD117 was performed in 30 cases, of which 29 (93.5\%) were positive and equivocal in $1(3.2 \%)$. DOG1 was performed in 16 cases, of them $15(48.4 \%)$ were positive and $1(3.2 \%)$ was negative [Figure 1]. CD34 was performed in 22 cases, of which $17(54.8 \%)$ were positive, while negative in 5 (16.1\%).

ASMA was performed in 16 cases, of which 7 (22.6\%) were positive and $9(29 \%)$ were negative. S100 was performed in 13 cases, of which $3(9.7 \%)$ were positive and $10(32.3 \%)$ were negative. CKAE1/AE3 was performed in only four cases, and all were negative. Desmin was performed in one case, which was negative.

Neoadjuvant chemotherapy was given in 11 cases $(35.5 \%)$. The mean duration of post-surgical followup was 30 months (range 4-59 months). Follow-up 
Table 1: Characteristics and risk stratification of patients

\begin{tabular}{|c|c|c|c|c|c|}
\hline No & Age (years) & Gender & Site & Morphological pattern & Risk stratification \\
\hline 1 & 59 & Male & Small intestine & Spindle cell & High risk \\
\hline 2 & 70 & Male & Extra visceral & Spindle cell & High risk \\
\hline 3 & 69 & Male & Stomach & Epithelioid & High risk \\
\hline 4 & 40 & Female & Rectum & Epithelioid & High risk \\
\hline 5 & 45 & Male & Extra visceral & Spindle cell & High risk \\
\hline 6 & 62 & Male & Extra visceral & Spindle cell & High risk \\
\hline 7 & 55 & Female & Extra visceral & Spindle cell & High risk \\
\hline 8 & 57 & Male & Stomach & Epithelioid & Intermediate risk \\
\hline 9 & 63 & Male & Jejunum/ileum & Mixed & High risk \\
\hline 10 & 23 & Female & Stomach & Epithelioid & Intermediate risk \\
\hline 11 & 53 & Male & Stomach & Spindle cell & High risk \\
\hline 12 & 65 & Male & Stomach & Spindle cell & Very low risk \\
\hline 13 & 68 & Male & Extra visceral & Epithelioid & High risk \\
\hline 14 & 68 & Male & Stomach & Spindle cell & Low risk \\
\hline 15 & 48 & Male & Stomach & Spindle cell & Low risk \\
\hline 16 & 20 & Female & Extra visceral & Spindle cell & Intermediate risk \\
\hline 17 & 72 & Male & Jejunum & Spindle cell & High risk \\
\hline 18 & & Female & Stomach & Spindle cell & High risk \\
\hline 19 & 49 & Male & Small intestine & Spindle cell & Intermediate risk \\
\hline 20 & 67 & Female & Stomach & Spindle cell & Low risk \\
\hline 21 & 58 & Female & Jejunum & Spindle cell & High risk \\
\hline 22 & 43 & Male & Duodenum & Epithelioid & High risk \\
\hline 23 & 45 & Female & Stomach & Spindle cell & Intermediate risk \\
\hline 24 & 41 & Male & Small intestine & Epithelioid & High risk \\
\hline 25 & 45 & Male & Stomach & Spindle cell & High risk \\
\hline 26 & 47 & Female & Stomach & Spindle cell & High risk \\
\hline 27 & 51 & Male & Small intestine & Spindle cell & High risk \\
\hline 28 & 54 & Male & Rectum & Mixed & High risk \\
\hline 29 & 62 & Female & Pancreas & Spindle cell & High risk \\
\hline 30 & 48 & Male & Stomach & Spindle cell & High risk \\
\hline 31 & 43 & Male & Duodenum & Spindle cell & High risk \\
\hline
\end{tabular}

was available in 21 patients. 7 patients (22.5\%) lost to follow-up. 8 (25\%) had recurrence and $4(12.9 \%)$ died.

\section{Discussion}

DeMatteo et al. reported GIST distribution to be $54 \%$ in stomach, $16 \%$ in rectum and $15 \%$ in small intestine. ${ }^{[1,10]}$ In DeMatteo et al. study, less than one-third of tumours were $\leq 5 \mathrm{~cm} .>2 / 3^{\text {rd }}$ of our tumours, i.e., $80.6 \%$ were $>5 \mathrm{~cm}$ in greatest tumour dimension. Postsurgically, negative margins were reported in $81 \%$ of cases. ${ }^{[10]}$ While we had negative resection margins in $51.6 \%$, probably due to large proportion of high-risk category GIST in our study. Follow-up in this study had a median follow-up of 24 months and documented disease-specific survival in $69 \%$ at 1 year. $46 \%$ were alive, $29 \%$ free of disease, $50 \%$ died of disease and $33 \%$ had isolated local recurrence. ${ }^{[10]}$

Zhao et al., 70\% of cases had spindle cell morphology and $20-25 \%$ had epithelioid while rest of cases had mixed appearance. Up to $95 \%$ of them are positive for CD117. GISTs can be negative or minimally positive for CD 117 in $<5 \%$ of cases. ${ }^{[6]}$ We had $58.1 \%$ of cases of spindle cell 


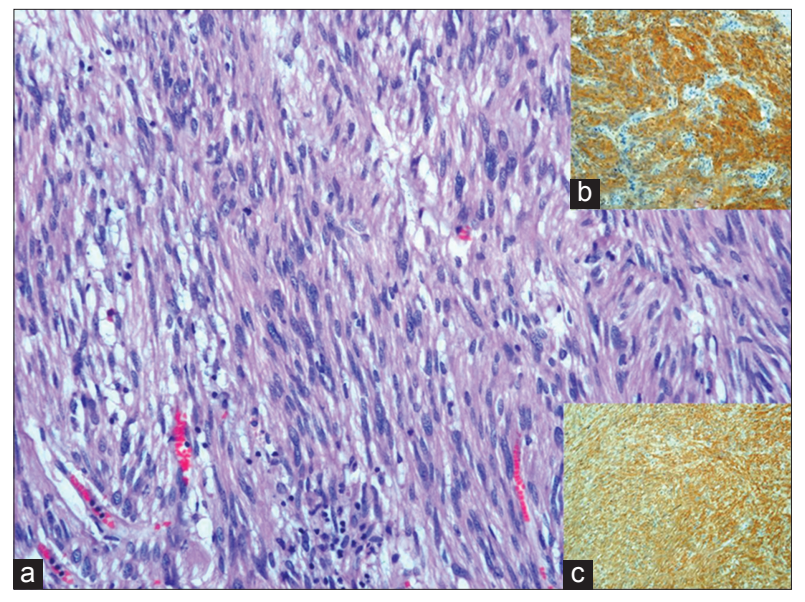

Figure 1: (a) $\mathrm{H}$ and $\mathrm{E}$ stained slide at $20 \mathrm{X}$ magnification showing spindle cells with mild polymorphism. (b) Inset shows IHC stain CD117 with diffuse immunoreactivity in tumour cells. (c) IHC stain DOG1 (discovered on GIST 1) showing diffuse positivity in tumour cells

morphology, $6.5 \%$ with epithelioid appearance and $6.5 \%$ had mixed morphology. The variation is likely due to nondocumentation of morphological appearance in nine cases which make up to $29.9 \%$ of them. CD117 was positive in $93.5 \%$ and equivocal in $3.2 \%$.

Miettinen et al. analysed 13 omental and 10 mesenteric GISTs with spindle cells or epithelioid cells; most of these tumours showed low mitotic activity. ${ }^{[11,12]}$ Reith et al. had analysed 48 GISTs of abdominal soft tissues, with the range of morphological features including purely epithelioid cells to those composed of spindle morphology as well some cases exhibiting mixed pattern. We had six extra-visceral cases, with four of them exhibiting spindle cell morphology while in two cases, pattern was not documented. Miettinen et al. had documented that omental and mesenteric GISTs were typically positive for CD117. ${ }^{[12]}$ Reith et al. tumours expressed CD117 in $100 \%{ }^{[13]}<5 \%$ of extraintestinal GIST are negative for CD117. ${ }^{[14]}$ All of our six cases were positive for CD117.

Data regarding survival of patients with adjuvant Imatinib therapy are limited. The median follow-up period was 54 months with observation that $92 \%$ of patients receiving 36 months of therapy were alive as compared to $82 \%$ alive for patients receiving 12 months. In recent larger institutional studies, a significant portion of GISTs had low to very low-risk chance of recurrence. ${ }^{[15-17]}$
Mucciarini et al. studied 124 GIST cases including $47 \%$ of high-risk cases. They observed 5-year disease-free survival rates after complete resection was $94 \%, 92 \%, 100 \%$ and $40 \%$ for patients at very low, low, intermediate and high risk, respectively. ${ }^{[3]}$ Of 31 analysed cases in our study, $71 \%$ GISTs were of high-risk category, $16.1 \%$ in intermediate risk and $9.7 \%$ in very low risk while $3.2 \%$ had very low risk. 21 patients $(67.7 \%)$ lost follow-up. 7 patients (22.5\%) are asymptomatic, $1(3.2 \%)$ had recurrence and $2(6.5 \%)$ died. One $(3.2 \%)$ of the asymptomatic patient received no further treatment after surgery. Patients who died and the one who had recurrence had GIST of high-risk group.

Surgical management of GISTs has been considered the most effective therapy. For locally advanced and metastatic GIST, Imatinib has been initiated. ${ }^{[17-20]}$ DeMatteo et al. concluded that although complete resection is associated with good outcome, it is not sufficient treatment. It had been observed that complete resection of GIST with adjacent organs, if required, should be performed. ${ }^{[10]}$

\section{Conclusion}

Majority of cases diagnosed at our centre were gastric in origin followed by small intestine, and as per risk categorisation, most were high risk. Patient survival with high-risk tumours was dismal.

\section{Competing Interest}

The authors declare that they have no competing interest.

\section{Conflict of Interest}

The authors declare that they have no conflict of interest.

\section{References}

1. Bashir U, Qureshi A, Khan HA, et al. Gastrointestinal stromal tumor with skeletal muscle, adrenal and cardiac metastases: An unusual occurrence. Indian J Pathol Microbiol 2011;54:362-4.

2. Greenson JK. Gastrointestinal stromal tumors and other mesenchymal lesions of the gut. Mod Pathol 2003;16:366-75.

3. Mucciarini C, Rossi G, Bertolini F, et al. Incidence and clinicopathologic features of gastrointestinal stromal tumors. A population-based study. BMC Cancer 2007;7:230.

4. Hemmings $C$, Yip D. The changing face of GIST: Implications for pathologists. Pathology 2014;46:141-8. 
5. Lv M, Wu C, Zheng Y, et al. Incidence and survival analysis of gastrointestinal stromal tumours in shanghai: A population-based study from 2001 to 2010 . Gastroenterol Res Pract 2014;2014:834136.

6. Jones RL. Practical aspects of risk assessment in gastrointestinal stromal tumors. J Gastrointest Cancer 2014;45:262-7.

7. Katie DL, Ivan D. Evaluating and reporting gastrointestinal stromal tumors after imatinib mesylate treatment. Open Path J 2009;3:53-7.

8. American Cancer Society. Gastrointestinal Stromal Tumour (GIST). American Cancer Society; 2015. Available from: http:// www.cancer.org cancer. [Last accessed on 2016 Aug 15].

9. Din OS, Woll PJ. Treatment of gastrointestinal stromal tumor: Focus on imatinib mesylate. Ther Clin Risk Manag 2008;4:149-62.

10. DeMatteo RP, Lewis JJ, Leung D, et al. Two hundred gastrointestinal stromal tumors: Recurrence patterns and prognostic factors for survival. Ann Surg 2000;231:51-8.

11. Miettinen M, Sobin LH, Lasota J. Gastrointestinal stromal tumors of the stomach: A clinicopathologic, immunohistochemical, and molecular genetic study of 1765 cases with long-term follow-up. Am J Surg Pathol 2005;29:52-68.

12. Miettinen M, Monihan JM, Sarlomo-Rikala M, et al. Gastrointestinal stromal tumors/smooth muscle tumors (GISTs) primary in the omentum and mesentery: Clinicopathologic and immunohistochemical study of
26 cases. Am J Surg Pathol 1999;23:1109-18.

13. Reith JD, Goldblum JR, Lyles RH, et al. Extragastrointestinal (soft tissue) stromal tumors: An analysis of 48 cases with emphasis on histologic predictors of outcome. Mod Pathol 2000; 13:577-85.

14. Qureshi A, Shiyam K, Sinan II, et al. C-kit negative extra intestinal gastrointestinal stromal tumour with no detectable mutations: A rare case. J Solid Tumors 2013;3:25-9.

15. Call J, Walentas CD, Eickhoff JC, et al. Survival of gastrointestinal stromal tumor patients in the imatinib era: Life raft group observational registry. BMC Cancer 2012;12:90.

16. Cheng Y, Zhang Z, Zhu H, et al. Clinicopathologic features of succinate dehydrogenase-deficient gastrointestinal stromal tumor. Zhonghua Bing Li Xue Za Zhi 2016;45:153-8.

17. Gao X, Xue A, Fang Y, et al. Role of surgery in patients with focally progressive gastrointestinal stromal tumors resistant to imatinib. Sci Rep 2016;6:22840.

18. Maghrebi H, Chebbi F, Makni A, et al. Laparoscopic resection of gastric stromal tumours. Tunis Med 2015;93:594-7.

19. Duman K, Harlak A. Independent prognostic factors and unsettled parameters of resected primary gastrointestinal stromal tumors. Surgery 2016;159:1696-7.

20. Li J, Zhang H, Chen Z, et al. Clinico-pathological characteristics and prognostic factors of gastrointestinal stromal tumors among a Chinese population. Int J Clin Exp Pathol 2015;8:15969-76. 